\title{
Die Lohnungleichheit von Vollzeitbeschäftigten in Deutschland: Rückblick und Überblick
}

\author{
Bernd Fitzenberger $\cdot$ Arnim Seidlitz
}

Online publiziert: 16 . Juli 2020

(C) Der/die Autor(en) 2020

Zusammenfassung In Westdeutschland stieg zwischen 1980 und 2010 die Lohnungleichheit von Vollzeitbeschäftigten deutlich an. Der Anstieg beschränkte sich zunächst auf den oberen Bereich der Lohnverteilung und setzte sich ab Mitte der 1990er Jahre sowohl im oberen als auch im unteren Bereich der Lohnverteilung fort. Im Zeitraum 1995 bis 2010 ging die Entwicklung mit starken Reallohnverlusten im unteren Bereich der Lohnverteilung einher. Nach 2010 stiegen die Reallöhne über die gesamte Lohnverteilung deutlich an, aber die Lohnungleichheit für Vollzeitbeschäftigte verblieb auf hohem Niveau trotz eines leichten Rückgangs am untersten Ende der Verteilung ab 2015. Dieser Beitrag dokumentiert und interpretiert die Entwicklung der Lohnungleichheit und geht auf mögliche Datenprobleme ein.

Schlüsselwörter Lohnungleichheit von Vollzeitbeschäftigten · Westdeutschland • SIAB-Daten

JEL-Klassifikation $\mathrm{J} 60 \cdot \mathrm{J} 31 \cdot \mathrm{C} 80$

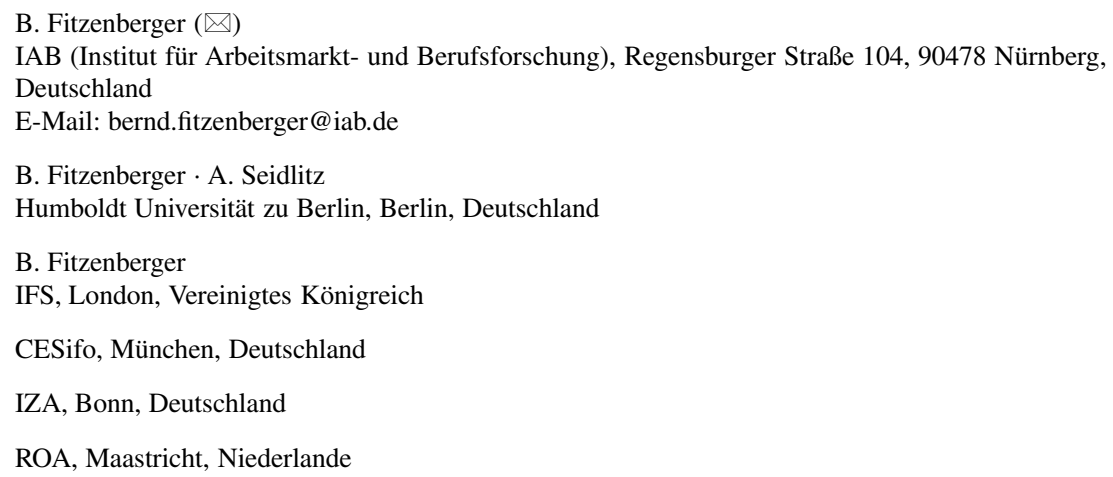




\section{Wage inequality among the full-time employed in Germany revisited: An overview}

Keywords Wage inequality of full-time employed $\cdot$ West-Germany $\cdot$ SIAB-data

Dieser Beitrag fasst die Inhalte der Heinz-Grohmann-Vorlesung zusammen, die Bernd Fitzenberger die Ehre hatte, anlässlich der Statistischen Woche 2019 in Trier zu halten. Wir danken den Zuhörerinnen und Zuhörern der Vorlesung, dem Herausgeber und einem/r Gutachter/in für hilfreiche Hinweise. Mit Martin Biewen, Mario Bossler, Christian Dustmann, Johannes Ludsteck, Joachim Möller, Dana Müller, Alexandra Spitz-Oener und Phillip vom Berge haben wir zu unterschiedlichen Zeitpunkten wertvolle Gespräche zu den Inhalten dieses Beitrages führen können. Wir danken Mario Bossler für die Zusammenarbeit bei den Analysen bis 2017, die dem Beitrag Bossler et al. (2020) entnommen sind. Teile dieses Beitrags basieren auf den früheren Überblicksaufsätzen Antonczyk et al. (2011) und Fitzenberger (2012). Dieser Beitrag ist Teil der Projekte „Female Employment Patterns, Fertility, Labor Market Reforms, and Social Norms: A Dynamic Treatment Approach“ (DFG-Projekt FI 692/14-1 und PA 2536/1-1) und „Accounting for Selection Effects in the Analysis of Wage Inequality in Germany“ (DFG-Projekt BI 767/3-1 und FI 692/161). Wir danken für die finanzielle Unterstützung durch die DFG. Alle Fehler diese Beitrages gehen alleine zu unseren Lasten.

\section{Einleitung}

Deutschland verzeichnete seit der Wiedervereinigung bis 2010 einen deutlichen Anstieg der Lohnungleichheit für Vollzeitbeschäftigte. In Westdeutschland begann die Ungleichheit sogar schon in den 1980er Jahren zu steigen. Zunächst beschränkte sich der Anstieg auf den oberen Bereich der Lohnverteilung. Ab Mitte der 1990er Jahre setzte sich der Anstieg sowohl im oberen als auch im unteren Bereich der Lohnverteilung fort. Diese Entwicklung wurde von einer umfangreichen Literatur auf Basis unterschiedlicher Datensätze und unterschiedlicher Analyseansätze dokumentiert. ${ }^{1}$ Nach 2010 stieg hingegen die Lohnungleichheit für Vollzeitbeschäftigte nicht weiter an, aber es kam auch nicht zu einem deutlichen Rückgang der Lohnungleichheit (Fitzenberger und Seidlitz 2020; Bossler et al. 2020). Der 2015 eingeführte gesetzliche Mindestlohn konnte die Ungleichheit nur im untersten Bereich der Lohnverteilung leicht reduzieren (Bossler et al. 2020).

Dieser Beitrag liefert einen systematischen Überblick der Entwicklung der Lohnungleichheit von Vollzeitbeschäftigten in Westdeutschland auf Basis der Ergebnisse von einschlägigen wissenschaftlichen Studien und ordnet die Entwicklung der

\footnotetext{
1 Siehe u. a. Fitzenberger (1999); Gernandt und Pfeiffer (2007); Dustmann et al. (2009, 2014); Antonczyk et al. (2010); Card et al. (2013); Möller (2016); Biewen und Seckler (2020); Bossler et al. (2020). Antonczyk et al. (2011) und Fitzenberger (2012) geben einen Überblick der Literatur bis Anfang der 2010er Jahre. Teile dieses Beitrags basieren auf diesen früheren Überblicksaufsätzen.
} 
Lohnungleichheit ein. Hierbei legen wir einen Schwerpunkt auf Probleme der verwendeten Datensätze für die Analyse der Lohnungleicheit.

Wir starten mit einem Rückblick zur Einordnung. Die Lohnungleichheit ist seit den 1980er Jahren in vielen industrialisierten Ländern angestiegen (Katz und Autor 1999; Machin und Van Reenen 2008). Noch zu Beginn des neuen Jahrtausends wurde argumentiert, dass ein solcher Anstieg der Lohnungleicheit in Westdeutschland nicht stattgefunden habe (Prasad 2004). Diese fehlende Flexibilität des deutschen Arbeitsmarktes wurde als nachteilig angesehen und zur Erklärung der damals hohen Arbeitslosigkeit herangezogen. Durch die Inflexibilität der Lohnstruktur würde selbst im Falle eines zyklischen Aufschwungs ein Abbau der Arbeitslosigkeit verhindert und deren Persistenz befördert. In internationalen Studien wurde von der unerträglichen Stabilität der deutschen Lohnstruktur gesprochen (Prasad 2004) und eine Senkung des Lohnniveaus sowie eine stärkere Flexibilisierung der Lohnstruktur wurden als notwendiges Mittel angesehen, damit Deutschland seine internationale Wettbewerbsfähigkeit und Anpassungsfähigkeit nicht verliere (Heckman 2002). Diese Literatur schrieb die fehlende Lohnflexibilität verschiedenen institutionellen Regelungen zu, wie beispielsweise Tarifverträgen, vergleichsweise hohen Ansprüchen auf Arbeitslosenunterstützung oder sonstige Sozialleistungen und Marktzutrittsbarrieren im Bereich von Produktmärkten oder beruflichen Arbeitsmärkten.

Die internationale Diskussion ignorierte dabei, dass schon vor der Mitte der 1990er Jahre ein Anstieg der Lohnungleichheit in Westdeutschland im oberen Bereich der Lohnverteilung zu beobachten war (Fitzenberger 1999; Dustmann et al. 2009), während gleichzeitig die Lohnungleichheit im unteren Bereich der Lohnverteilung bis Anfang der 1990er Jahre nahezu konstant blieb. Diese Entwicklungen standen im Einklang mit der Annahme, dass der institutionelle Rahmen in dieser Zeit eine Flexibilisierung im Niedriglohnbereich und in der Folge einen Anstieg der Ungleichheit im Bereich niedriger Löhne noch verhindert hat (siehe Fitzenberger 1999 und Antonczyk et al. 2018 für diese Interpretation). Schon vor Mitte der 1990er Jahre entwickelten sich jedoch die Löhne im oberen Bereich der Lohnverteilung auseinander.

Inzwischen belegt eine umfangreiche Literatur einen im internationalen Vergleich sehr deutlichen Anstieg der Lohnungleichheit im unteren Bereich der Lohnverteilung und eine Fortsetzung des Anstiegs im oberen Bereich der Lohnverteilung seit der ersten Hälfte der 1990er Jahre bis zum Jahr 2010 (siehe u. a. Dustmann et al. 2009; Gernandt und Pfeiffer 2007; Antonczyk et al. 2018; Card et al. 2013). In den 2000er Jahren ging dieser Anstieg der Ungleichheit mit einem Rückgang der Reallöhne in der unteren Hälfte der Lohnverteilung einher (Fitzenberger und Seidlitz 2020). Dieser Verlust der Kaufkraft von Niedriglohnbeschäftigten setzte sich bis etwa 2012 fort.

In der öffentlichen Wahrnehmung genießen das gestiegene Niveau der Lohnungleichheit und der Rückgang der realen Arbeitseinkommen seit Jahren eine besondere Aufmerksamkeit. Diese Entwicklung war eine wesentliche Motivation für die Einführung des gesetzlichen Mindestlohns im Jahr 2015. Bossler und Schank (2020) untersuchen die Wirkung des Mindestlohns auf die Entwicklung der Lohnungleichheit von Vollzeitbeschäftigten in Deutschland (siehe auch Bossler et al. 2020). Die Ergebnisse der Studie besagen, dass der Mindestlohn die Lohnungleichheit am un- 
teren Rande etwas reduzieren konnte. Allerdings fingen die Reallöhne auch schon vor Einführung des Mindestlohns ab 2012 wieder zu steigen an. Die Ungleichheit wurde in den 2010er Jahren jedoch kaum reduziert. Weil die Löhne im unteren und oberen Bereich der Verteilung in etwa der gleichen Rate stiegen, blieb sie stabil. Auch lagen die Reallöhne 2017 am unteren Teil der Verteilung für Frauen nur in etwa auf dem Niveau von 2000 und für Männer sogar darunter (Bossler et al. 2020).

In der Literatur werden verschiedene Hypothesen zur Erklärung des Anstiegs der Lohnungleichheit im unteren Bereich der Lohnverteilung diskutiert. Ein Erklärungsansatz beruht auf Veränderungen in der Arbeitsnachfrage ausgelöst durch technischen Fortschritt. Die ursprüngliche Version geht dabei davon aus, dass sich die Arbeitsnachfrage nach hochqualifizierten gegenüber der Arbeitsnachfrage nach niedrigqualifizierten Beschäftigten gleichförmig über die gesamte Lohnverteilung hinweg erhöht (,Skill Bias in Labor Demand“, Katz und Autor 1999). Eine Weiterentwicklung dieser Erklärung ist der sogenannte tätigkeitsbasierte Ansatz (,TaskBased Approach“). Er misst der Substitution von Routine-Tätigkeiten durch Computer und Maschinen eine zentrale Bedeutung bei (siehe Acemoglu und Autor 2011 für einen Überblick und Spitz-Oener 2006 für eine grundlegende Analyse zu Deutschland). Im Gegensatz zu einer gleichgerichteten Verzerrung der Technologieeffekte in allen Bereichen der Lohnverteilung erlaubt es der tätigkeitsbasierte Ansatz eine U-förmige Polarisierung der Beschäftigung zu erklären, wie sie seit den 1990er Jahren in den USA, Großbritannien und Deutschland beobachtet wird (SpitzOener 2006; Dustmann et al. 2009). Demnach steigt sowohl die Beschäftigung von Hochqualifizierten als auch von Niedrigqualifizierten relativ zu der Beschäftigung von Personen mit mittlerem Qualifikationsniveau an, was in der Folge auch eine U-förmige Lohnentwicklung über die Lohnverteilung impliziert.

Dustmann et al. (2009); Fitzenberger (1999) und Antonczyk et al. (2018) zeigen, dass der technologische Forschritt durchaus zur Entwicklung der Ungleichheit im oberen Bereich der Einkommensverteilung beiträgt. Der tätigkeitsbasierte Ansatz kann auf der anderen Seite einen Teil des Anstiegs der Ungleichheit für Geringverdienende begründen (Antonczyk et al. 2009). Letztlich scheinen neue Technologien jedoch nicht alleine die Lohnentwicklung zu erklären, ansonsten würden sich die Veränderungen in unterschiedlichen Ländern, wie den USA und Deutschland, nicht so stark unterscheiden (Dustmann et al. 2009; Antonczyk et al. 2018).

Den Arbeitsmarktreformen zu Beginn des neuen Jahrtausends (insbesondere den sogenannten Hartz-Reformen), welche die Flexibilität des Arbeitsmarktes erhöhen sollten, wird häufig ein Einfluss auf die Lohnentwicklung zugeschrieben. Das gilt hauptsächlich für den Anstieg der Lohnungleichheit im unteren Bereich der Lohnverteilung - z. B. wäre ein Sinken der niedrigen Löhne als Reaktion auf Kürzungen der Lohnersatzleistungen für Arbeitslose denkbar. Jedoch setzte der Anstieg der Ungleichheit spätestens Mitte der 1990er Jahre, also etwa zehn Jahre vor den HartzReformen, ein. Obwohl diese somit nicht als Auslöser des Anstieges der Lohnungleichheit angesehen werden können, ist es plausibel zu unterstellen, dass das Wachstum des Niedriglohnsektors durch die Arbeitsmarktreformen befördert wurde und ab Mitte der 2000er Jahre so auch zur wachsenden Ungleichheit beigetragen hat (Dustmann et al. 2014). 
Ein weiterer Literaturstrang betont die potenzielle Bedeutung der Mobilität im Arbeitsmarkt (Riphahn und Schnitzlein 2016). Zunehmende Unterschiede in der Job-Stabilität oder in den Verdienstchancen eines neuen Jobs können zu einem Anstieg der Lohnungleichheit führen. In Anlehnung an diese Argumentation betont die Studie von Card et al. (2013) die Bedeutung der Firmenunterschiede in der Lohnsetzung. Auch wenn Firmen nicht tarifgebunden sind, setzen sie eine vergleichsweise einheitliche Lohnpolitik für alle Beschäftigten um. Dadurch entstehen Lohnunterschiede zwischen ansonsten gleichen Beschäftigten, die bei unterschiedlichen Firmen arbeiten. Card et al. (2013) argumentieren, dass die Entlohnungsunterschiede zwischen den Firmen in Deutschland zugenommen haben und diese Entwicklung eine Haupterklärung für den Anstieg der Lohnungleichheit in Deutschland darstelle.

In der Literatur besteht ein hoher Konsens darüber, dass Verzerrungen im technischen Fortschritt zu einer Erhöhung der Lohnungleichheit der Vollzeitbeschäftigten in Westdeutschland geführt haben. Kontroverser wird die Bedeutung der drei folgenden Erklärungsansätze diskutiert: (1) Rückgang der Tarifbindung, (2) Zunahme der Heterogenität der Beschäftigten, (3) Phasen steigender und fallender Arbeitslosigkeit. Auf diese Erklärungsansätze, zu denen aktuelle Befunde vorliegen, gehen wir in diesem Beitrag vertieft ein.

Im Weiteren gehen wir wie folgt vor: Zunächst erfolgt in Abschn. 2 eine zusammenfassende Darstellung und Diskussion der Entwicklung der Lohnungleichheit auf Basis der einschlägigen Literatur. Abschn. 3 beschreibt die SIAB-Daten einschließlich des Strukturbruchs in der Erfassung der Vollzeiterwerbstätigkeit im Jahr 2011. Es erfolgt eine Gegenüberstellung der Entwicklung der Lohnungleichheit in Westdeutschland zwischen 2000 und 2017 mit und ohne Korrektur des Strukturbruchs. Abschn. 4 diskutiert, ob und inwieweit die dargestellen empirischen Ergebnisse die unterschiedlichen ökonomischen Hypothesen stützen. Abschn. 5 fasst die zentralen Ergebnisse und noch offene Forschungsfragen zusammen.

\section{Literatur zur Entwicklung der Lohnungleichheit}

Dieser Abschnitt fasst die empirischen Ergebnisse in einem selektiven Überblick der umfangreichen einschlägigen Literatur zur Entwicklung der Lohnungleichheit in Deutschland zusammen.

$\mathrm{Zu}$ Beginn ist darauf hinzuweisen, dass sich die deskriptiven Analysen auf die Lohnentwicklung je nach betrachteter Gruppe deutlich unterscheiden können. Ergebnisse können variieren je nach dem, ob Männer und/oder Frauen, ob West- und/ oder Ostdeutschland und Vollzeitbeschäftigte und/oder Teilzeitbeschäftigte betrachtet werden. Ferner ist es in der Literatur üblich, sich bezüglich dem Alter auf einen Kernbereich zu beschränken. Oft werden nur Beschäftigte, welche älter als 20 bis 25 Jahre und jünger als 50 bis 60 Jahre sind, mit eingeschlossen. Unterschiede in diesen Einschränkungen der Daten können die Vergleichbarkeit der verschiedenen Studien beeinflussen.

Die in der Forschungsliteratur berichteten Ergebnisse beziehen sich meist auf Vollzeiterwerbstätige in Westdeutschland, häufig nur auf Männer. Der größere Teil der Literatur verwendet die Paneldaten des SIAB, welche auf den Integrierten Er- 
werbsbiographien des Instituts für Arbeitsmarkt- und Berufsforschung (IAB) basieren (SIAB7517, vgl. Antoni et al. 2019 für die aktuelle Version dieser Daten). Ein Nachteil dieser Daten ist jedoch, dass die Verdienste nicht auf Stunden-, sondern auf Tagesebene angegeben werden. Teilzeitbeschäftigte bleiben deswegen bei Analysen der SIAB-Daten außen vor, weil Unterschiede in ihren Verdiensten sowohl von unterschiedlich hohen Löhnen als auch unterschiedlich langen Arbeitszeiten, die nicht erhoben werden, verursacht sein können. Im Absch. 3 wird intensiver auf die SIABDaten eingegangen.

Bevor wir auf die einzelnen Studien eingehen, ist schließlich ein methodischer Hinweis angebracht. Die Mehrzahl der Studien zur Entwicklung der Lohnungleichheit betrachtet als Ungleichheitsmaß die Differenzen verschiedener Quantile der logarithmierten Löhne. ${ }^{2}$ Für die Interpration ist dabei interessant, dass kleine Veränderungen der Differenz von logarithmierten Löhnen in etwa der relativen Veränderung des Verhältnisses der Löhne (nicht-logarithmiert) in Prozent entsprechen. Unterstellen wir zum Beispiel einen Anstieg der Differenz zwischen dem 85\%-und dem 15\%-Quantil der logarithmierten Löhne um 0,15. Dann wird dieser Anstieg um 0,15 auch als ein Anstieg um 15 Logpunkte bezeichnet und entpricht einem Anstieg des Verhältnisses zwischen dem 85\%- und dem 15\%-Quantil um 16 Prozent, da $\exp (0,15) \approx 1,16=116 \%$.

Die Literatur zur Entwicklung der Lohnungleichheit betrachtet die Ungleichheit der logarithmierten Löhne, da Löhne als Preise des Faktors Arbeit aufzufassen sind und die Arbeitsnachfrage der Betriebe nach verschiedenen Gruppen von Beschäftigten eine Funktion der relativen Löhne ist (Fitzenberger 1999). Die Betrachtung der Quantilsdifferenzen erlaubt es in einfacher Weise, die Entwicklung der Ungleichheit in verschiedenen Bereichen der Lohnverteilung getrennt zu betrachten. Einige Studien verwenden die Varianz der logarithmierten Löhne. Weitere Vorteile der Betrachtung der Quantile sind ihre Robustheit genüber Ausreisern und gegenüber einer Rechtszensierung der Löhne wie in den SIAB-Daten, solange das betrachtete Quantil unterhalb der Zensierungsgrenze liegt (Fitzenberger 1999). Aus den genannten Gründen werden in der Literatur zur Entwicklung der Lohnungleichheit sehr selten summarische Ungleichheitsmaße - wie der Gini-Koeffizient - verwendet.

Unser Literaturüberblick startet mit Gernandt und Pfeiffer (2007). Die Studie verwendet das Sozio-oekonomische Panel ${ }^{3}$ zur Untersuchung der Entwicklung der Lohnungleicheit in Deutschland zwischen 1984 und 2005 für männliche Beschäftigte im Alter zwischen 25 und 55 Jahren in Westdeutschland. Laut der Studie steigt die Streuung der Löhne, gemessen am Verhältnis des 90\%-Quantils zum 10\%-Quantil, von 2,5 auf 3,1 in Westdeutschland und in Ostdeutschland noch stärker von 2,4 auf 3,2 .

\footnotetext{
2 Unter dem x\%-Quantil versteht man die Lohnhöhe, bei der maximal x\% der Beschäftigten einen Lohn unterhalb dieses Niveaus und maximal $(100-x) \%$ der Beschäftigten einen Lohn oberhalb dieses Niveaus aufweisen. Dies bedeutet bspw., dass in etwa $10 \%$ weniger als das $10 \%$-Quantil der Löhne und in etwa 90\% mehr als das 10\%-Quantil der Löhne verdienen. Der Median als 50\%-Quantil teilt die Lohnverteilung in die $50 \%$ der Beschäftigten, die weniger als den Median verdienen, und die $50 \%$ der Beschäftigten, die mehr als den Median verdienen. Da der Logarithmus eine streng monotone steigende Funktion ist, entspricht der Logarithmus eines Lohnquantils dem entsprechenden Quantil des logarithmierten Lohns.

3 Eine jährliche Haushaltsbefragung seit 1984 in Westdeutschland und seit 1990 in Ostdeutschland.
} 
Fitzenberger (1999) zeigt auf Basis von Daten der IAB-Beschäftigtenstichprobe 1975 bis 1990 - diese Daten sind eine frühere Version des SIABs -, dass die Lohnungleichheit in Westdeutschland im oberen Bereich der Lohnverteilung in den 1980er Jahren zugenommen hat. Antonczyk et al. (2018) verwenden die IAB-Beschäftigtenstichprobe bis 2004 im Rahmen eines Vergleichs der Entwicklung der Lohnungleichheit zwischen Westdeutschland und den USA. Für die westdeutschen Männer zwischen 25 und 55 Jahren zeigt sich seit Beginn der 1990er Jahr ein kontinuierlicher Anstieg der Lohnungleichheit im unteren Bereich der Lohnverteilung (Differenz Median und 20\%-Quantil), der sich mit dem Ende der 1990er Jahre beschleunigt. Für niedrig- und mittelqualifizierte Beschäftigte im unteren Bereich der Lohnverteilung fällt sogar seit den 1990er Jahren der Reallohn. Die weitergehenden Analysen in Antonczyk et al. (2018) zeigen, dass insbesondere die Niedrigverdienenden in den jungen Kohorten von Arbeitnehmern mit niedriger und mittlerer Qualifikation besonders starke Reallohnverluste seit Mitte der 1990er Jahre erfahren mussten. Hierin zeigt sich das Entstehen des Niedriglohnsektors in Westdeutschland. Die Zusammensetzung der Kohorten im Arbeitsmarkt erklärt einen kleineren Teil des Anstiegs der Lohnungleichheit in Westdeutschland (Antonczyk et al. 2018), vor allem im Hinblick auf die Lohnungleichheit im unteren Teil der Lohnverteilung.

In einer viel beachteten Studie untersuchen Dustmann et al. (2009) die Entwicklung der Lohnstruktur für vollerwerbstätige Männer und Frauen in Westdeutschland ebenfalls auf Basis der IAB-Beschäftigtenstichprobe bis 2004. Sie zeigen, dass von 1990 bis 2004 die Differenz zwischen dem 85\%-Quantil und dem 15\%-Quantil sowohl für Männer als auch für Frauen um über 15 Logpunkte anstieg. Ähnlich wie Antonczyk et al. (2018) zeigen Dustmann et al. (2009), dass Kompositionsveränderungen einen Teil des Anstiegs der Lohnungleichheit erklären können, allerdings betrifft dies vor allem den Anstieg der Lohnungleichheit im oberen Bereich der Verteilung.

Riphahn und Schnitzlein (2016) untersuchen den Rückgang der Lohnmobilität (Wechsel der Position in der Lohnverteilung) in Ost- und Westdeutschland auf Basis der SIAB-Daten bis 2008. Sie belegen, dass sich der Anstieg der Lohnungleichheit im oberen und im unteren Bereich der Lohnverteilung zwischen 2004 und 2008 analog zu den Ergebnissen in Antonczyk et al. (2018) und Dustmann et al. (2009) fortsetzt. Auch der Rückgang der Reallöhne am 20\%- und 50\%-Quantil scheint sich nach 2004 noch leicht zu beschleunigen. Die Ergebnisse in Riphahn und Schnitzlein (2016) bedeuten, dass in Folge des Rückgangs der Lohnmobilität die erreichte relative Lohnposition persistenter geworden. Somit lasse sich aus dem Anstieg der Lohnungleichheit nicht auf eine Zunahme der Flexibilität des Arbeitsmarkts schlieBen.

Card et al. (2013) untersuchen die Bedeutung der Firmenheterogenität für den Anstieg der Lohnungleichheit für vollzeiterwerbstätige Männer im Alter von 20 bis 60 Jahren in Westdeutschland. Die Studie verwendet die kompletten Daten der Beschäftigtenstatistik von 1985 bis 2008. Die Studie belegt auf einer umfassenderen Datenbasis als alle bisherigen Studien den Anstieg der Lohnungleichheit bis 2009. Insgesamt findet die Studie einen kontinuierlichen Anstieg der Lohnungleichheit im oberen und unteren Bereich der Lohnverteilung seit Mitte der 1980er Jahre. Card et al. (2013) zeigen weiterhin, dass ein großer Teil des Anstiegs der Lohnungleich- 
heit innerhalb von Gruppen mit gleichem Ausbildungsniveau und Berufserfahrung erfolgt, d.h. nicht das Ergebnis von Effekten der Veränderung in der Komposition der Beschäftigten ist. Als zentrales Ergebnis zeigt die Studie, dass ein großer Teil des Anstiegs der Lohnungleichheit auf die zunehmende Firmenheterogenität zurückgeführt werden kann.

Goldschmidt und Schmieder (2017), die die gleiche Datenbasis verwenden, untersuchen die Bedeutung von Outsourcing von Dienstleistungen aus größeren Betrieben - die Verlagerung von Tätigkeiten in der Gebäudereinigung, Sicherheit, Logistik und dem Catering an Subunternehmen. Die Studie zeigt, dass die Löhne der Beschäftigten durch das Outsourcing um 10 Prozent sinken. Da es sich hauptsächlich um ohnehin eher niedrig entlohnte Dienstleistungen handelt, kann das Outsourcing einen Teil des Anstiegs der Lohnungleichheit in der unteren Hälfte der Verteilung erklären. Zum Anstieg in der oberen Hälfte trägt es nicht wesentlich bei.

Die Analyse des Anstiegs der Lohnungleichheit für vollzeiterwerbstätige Männer und Frauen in der Studie Antonczyk et al. (2010) basiert auf den Verdienststrukturerhebungen des Statistischen Bundesamtes für die Jahre 2001 und 2006 für Westdeutschland. Diese Daten erlauben es im Gegensatz zu den Studien, die auf der Beschäftigtenstatistik oder den SIAB-Daten basieren, Stundenlöhne zu berechnen. Ein Fokus der Analyse liegt auf der Frage, welchen Beitrag der starke Rückgang der Tarifbindung für den Anstieg der Lohnungleichheit aufweist. Die Studie zeigt, dass das Verhältnis zwischen dem 90\%- und 10\%-Quantil für Männer um 13 Logpunkte und für Frauen um 11 Logpunkte innerhalb eines Zeitraums von nur fünf Jahren angestiegen ist, wobei der Anstieg im unteren Bereich der Lohnverteilung stärker als im oberen Bereich der Lohnverteilung ausfällt. Der Anstieg der Lohnungleichheit geht mit Reallohngewinnen im oberen Bereich der Lohnverteilung und mit Reallohnverlusten unterhalb des Medians einher. Weiterhin zeigt sich, dass der beobachtete starke Rückgang der Tarifbindung (minus 17 Prozentpunkte für Männer und minus 19 Prozentpunkte für Frauen) zu einem kleineren Teil zum Anstieg der Lohnungleichheit beiträgt. Gleichzeitig steigt jedoch die Lohnungleichheit innerhalb der Regime der Tarifbindung stark an und die Effekte der Tarifbindung werden durch die Veränderungen der Wirkungen von firmenspezifischen Variablen auf die Verteilung der Löhne dominiert. Letztere können nahezu komplett durch Brancheneffekte erklärt werden. Die hohe Bedeutung der Firmenebene für die Erklärung des Anstiegs der Lohnungleichheit ähnelt den oben erwähnten Ergebnissen in Card et al. (2013), allerdings beruhen die Ergebnisse auf sehr unterschiedlichen Analyseverfahren.

Antonczyk et al. (2009) untersuchen den Anstieg der Lohnungleichheit für vollzeiterwerbstätige Männer in Westdeutschland auf Basis der Erhebungen „Qualifikation und Berufsverlauf“ und „Erwerbstätigenbefragung“ des Bundesinstituts für Berufsbildung (BIBB) in den Jahren 1999 und 2006, welche ebenfalls eine Analyse der Stundenlöhne ermöglichen. In diesen Umfragedaten werden explizit die Tätigkeiten auf individueller Ebene erfasst. Die Studie untersucht, inwiefern ein tätigkeitsbasierter Ansatz zur Erklärung des Anstiegs der Lohnungleichheit beiträgt. Sie ermittelt einen Anstieg des Verhältnisses zwischen dem 80\%- und 20\%-Quantil der Löhne für Männer um knapp 8 Logpunkte, wobei der Anstieg im unteren Bereich der Lohnverteilung etwas stärker als im oberen Bereich ausfällt. Es zeigt sich, 
dass ein tätigkeitsbasierter Ansatz den Anstieg der Lohnungleichheit in Deutschland nicht erklären kann.

In einer aktuellen Studie für Gesamtdeutschland nutzen Biewen und Seckler (2020) ebenfalls verschiedene Wellen der Verdienststrukturerhebung bis 2010. Im Gegensatz zu Antonczyk et al. (2010) identifiziert die Studie den Rückgang der Tarifbindung als wichtigsten Faktor für den Anstieg der Lohnungleichheit. Weitere wichtige Erklärungsfaktoren sind Veränderungen im Alter und der Bildung der Beschäftigten. Es ergeben sich nur schwache Effekte des internationalen Handels, der Heterogenität von Firmen, der Veränderung von Tätigkeiten und der regionalen Konvergenz zwischen Ost- und Westdeutschland. Die Analyse in Biewen und Seckler (2020) ist mit der Studie Antonczyk et al. (2010) aus zwei Gründen schwer vergleichbar. Zum einen beschränkt sich letztere auf Westdeutschland und zum anderen verändert sich über die verschiedenen Wellen der Verdienststrukturerhebung die Abgrenzung der erfassten Branchen, was längerfristige Analysen erschwert.

Einen Blick über das Jahr 2010 hinaus, nämlich bis 2014, werfen Baumgarten et al. (2020); Möller (2016) und Fitzenberger und Seidlitz (2020). Wichtigster Befund dabei ist, dass eine Trendwende festzustellen ist, dahingehend dass die Ungleichheit in den 2010er Jahren nicht weiter ansteigt. Die drei Studien beruhen alle auf Datensätzen, welche auf der Beschäftigtenstatistik (SIAB-Daten des IAB) basieren. Die Daten deuten darauf hin, dass sich für Frauen die Ungleichheit im unteren Bereich der Lohnverteilung sogar leicht verringert haben könnte (Möller 2016 und Fitzenberger und Seidlitz 2020), gleiches gilt für Ostdeutschland (Möller 2016). Eine generelle Trendumkehr hin zu einer niedrigeren Lohnungleichheit ist jedoch nicht festzustellen. Die Ungleichheit scheint bei seit 2011 real ansteigenden Löhnen weitestgehend stabil zu sein. In den Analysen von Baumgarten et al. (2020) und Möller (2016) sind es die Unterschiede zwischen den Firmen, welche auf den Verlauf der Ungleichheit - Anstieg und anschließende Stagnation - den stärksten Einfluss haben.

Unter Verwendung der gleichen Datenbasis bis 2017 zeigen Bossler et al. (2020), dass im untersten Bereich der Verteilung die Lohnungleichheit ab 2012 leicht zurück ging. Wenn alle Beschäftigten in Gesamtdeutschland betrachtet werden - Frauen und Männer in Ost- und Westdeutschland - nahm das 10-Prozent Quantil seit 2012 um über 11 Logpunkte zu, während die 20-, 50- und 80-Prozent Quantile höchstens um 9 Logpunkte zunahmen. Der Mindestlohn, welcher 2015 eingeführt wurde, kann den über den Rest der Lohnverteilung hinausgehenden Anstieg des 10-Prozent Quantils zu einem erheblichen Teil erklären, während er auf die Entwicklung der Medianlöhne keinen Einfluss hatte. Dieser ungleichheitsreduzierende Effekt lässt sich auf Ostdeutschland zurückführen (Bossler und Schank 2020). Wenn nur westdeutsche Vollzeitlöhne betrachtet werden, hat der Mindestlohn keinen messbaren Effekt auf die Lohnungleichheit (Bossler et al. 2020).

Brüll und Gathmann (2019) untersuchen die Entwicklung der Lohnungleichheit in Ostdeutschland auf Basis der SIAB-Daten bis 2014. Im Fokus der Analyse stehen der Einfluss zweier institutioneller Änderungen, des Rückgangs der Tarifbindung und der Einführung branchenspezifischer Mindestlöhne, sowie des auswanderungsbedingten Rückgangs im Angebot hochqualifizierter Beschäftigter. Während letzteres einen bedeutenden Teil des Anstiegs der Lohnungleichheit im oberen Bereich der 
Verteilung erklärt, sind die beiden institutionellen Änderungen starke Treiber des Anstiegs der Lohnungleichheit im unteren Bereich der Lohnverteilung.

An dieser Stelle muss darauf hingewiesen werden, dass alle Studien - mit Ausnahme von Möller (2016) und Studien, an denen die Autoren dieses Artikels beteiligt sind -, die die SIAB-Daten (oder andere Daten aus der Beschäftigtenstatistik) ab 2011 verwenden, nicht dem Strukturbruch in der Erfassung des Vollzeitstatus Rechnung tragen. Dieser Strukturbruch kann potenziell die Ergebnisse beeinflussen und hierauf wird in Abschn. 3 einzugehen sein.

Zusammenfassend lässt sich sagen, dass die Literatur einen ausgeprägten Anstieg der Lohnungleichheit für Männer und Frauen in West- und Ostdeutschland spätestens seit Mitte der 1990er Jahre bis etwa 2010 belegt. Der Anstieg erfolgt im oberen und im unteren Bereich der Lohnverteilung. Seit Mitte der 1990er Jahre geht er mit Reallohnverlusten in der Mitte und im unteren Bereich der Lohnverteilung - vor allem in den 2000er Jahren - einher. Ein großer Teil des Anstiegs der Lohnungleichheit wird innerhalb von Berufserfahrungs-Qualifikations-Gruppen (oder Alters-Qualifikations-Gruppen) beobachtet und geht somit nicht auf Kompositionseffekte zurück.

\section{Strukturbruch in SIAB-Daten und Lohnungleichheit seit 2000}

In Ergänzung zur vorausgegangenen Literaturübersicht wollen wir in diesem Abschnitt nochmals einen vertieften Blick auf die Entwicklung der Lohnungleichheit zwischen 2000 und 2017 werfen. Hierzu wird die Stichprobe der Integrierten Arbeitsmarktbiografien (SIAB-Daten) vom Institut für Arbeitsmarkt- und Berufsforschung (Antoni et al. 2019) verwendet, welche der wichtigste und meist genutzte Datensatz für Analysen des deutschen Arbeitsmarktes ist. ${ }^{4}$ Im Folgenden gehen wir auf den schon oben erwähnten Strukturbruch ein und beschreiben das von uns in Fitzenberger und Seidlitz (2020) entwickelte Korrekturverfahren.

\subsection{SIAB-Daten und Strukturbruch 2011}

Die SIAB-Daten werden vom Institut für Arbeitsmarkt- und Berufsforschung (IAB) bereit gestellt und umfassen in ihrer aktuellen Version den Zeitraum bis 2017. Wir nutzen die anonymisierte Version dieser Daten, die „Regionalfiles“.

Bei den Daten handelt es sich um eine Zwei-Prozent-Stichprobe der integrierten Erwerbsbiographien (IEB), einer Aufbereitung der Beschäftigtenstatistik am IAB. Die Datenbasis liegt seit 1975 für West- und seit 1992 für Ostdeutschland vor und enthält neben Informationen über den Bezug von Lohnersatzleistungen für alle sozialversicherungspflichtigen Beschäftigten Informationen zu ihrer Beschäftigung.

\footnotetext{
4 Von den oben genannten Studien stützen sich Fitzenberger (1999); Dustmann et al. (2009); Möller (2016); Riphahn und Schnitzlein (2016); Antonczyk et al. (2018); Brüll und Gathmann (2019); Baumgarten et al. (2020) und Fitzenberger und Seidlitz (2020) auf frühere oder verwandte Versionen dieser Daten. Card et al. (2013) und Goldschmidt und Schmieder (2017) nutzen die kompletten Daten der Beschäftigungsstatistik, von denen die Daten der anderen Studien eine Stichprobe sind. Bossler et al. (2020) und Bossler und Schank (2020) beruhen auf der gleichen Datenbasis.
} 
Diese umfassen Angaben zum Geschlecht, Bildungsabschlüssen, Teilzeitstatus und Berufskategorie der beschäftigten Person, der Industriekategorie und der Region des Betriebs, die tagesgenaue Dauer des Beschäftigungsverhältnisses und den Bruttotageslohn. Keine Daten liegen vor über Personen, die nicht sozialversicherungspflichtig beschäftigt oder Lohnersatzleistungen der Bundesagentur für Arbeit beziehen. Dies sind vor allem Selbstständige und Beamte. Geringfügig Beschäftigte werden erst seit 1999 erfasst.

Die Daten bilden nur unzureichend die Arbeitszeiten ab. Über den Teilzeitstatus hinaus gibt es eine keine Information über Arbeitsstunden. Das führt dazu, dass alle Studien in Fußnote 4 generell nur Vollzeiterwerbstätige betrachten. Dabei stützen sie sich auf die Annahme, dass für Menschen in Vollzeit die Arbeitszeit ähnlich ist und konstant bleibt. Veränderungen in der Entlohnung sind somit immer auf Änderungen im Stundenlohn zurückzuführen. Für Teilzeitbeschäftigte ist dieser Rückschluss so nicht möglich, weil sich für sie auch die in Daten nicht vorhandenen Arbeitsstunden ändern könnten. Und für Vollzeitbeschäftigte ignoriert diese Annahme die Variation der Verdienste aufgrund unterschiedlicher Arbeitszeiten durch Unterschiede in der Normalarbeitszeit oder Überstunden.

Der Ansatz, sich nur auf die Vollzeitbeschäftigten zu beschränken, beruht auf einer genauen Identifikation von Vollzeit- und Teilzeitbeschäftigungsverhältnissen. Leider zeigt der Indikator für diese in den SIAB-Daten auffällige Inkonsistenzen. 2011 wurde das System der Beschäftigungsmeldungen reformiert. Dabei stieg der Anteil an Teilzeitbeschäftigten zwischen 2010 und 2012 sprunghaft an. Es offenbarte sich, dass in den Jahren zuvor offensichtlich Teilzeitbeschäftigte fälschlicherweise als Vollzeitkräfte angegeben wurden. Fitzenberger und Seidlitz (2020) widmen sich ausführlich diesem Problem und entwickeln ein Korrekturverfahren.

Durch die fehlerhafte Erfassung des Teilzeitstatus in den Daten bis 2011 entsteht das Problem, dass die Quantile der Lohnverteilung nach unten hin verzerrt werden. Der Grund hierfür ist, dass das Tagesentgelt der Teilzeitkräfte aufgrund der kürzeren Arbeitszeit geringer ausfällt. Diese Verzerrung variiert über die Lohnverteilung, sprich niedrige Quantile werden stärker nach unten verzerrt als höhere. Somit wird das Niveau der Lohnungleichheit überschätzt. Für die Analyse über die Zeit kann das zu groben Fehleinschätzungen führen. Auf den ersten Blick sieht es in den SIAB-Daten so aus, als ob sich die Lohnungleichheit (unter Vollzeitbeschäftigten) zwischen 2010 und 2012 stark reduziert. Aufgrund des Strukturbruchs läge hier jedoch ein Trugschluss vor. 2010 sind in den Vollzeitbeschäftigungsverhältnissen noch die fehlerhaften Teilzeitbeschäftigten enthalten, welche die Ungleichheit fälschlicherweise als höher erscheinen lassen. 2012 ist die Identifikation der Teilzeitbeschäftigten zuverlässiger. Die Verringerung der Lohnungleichheit zwischen 2010 und 2012 ist nahezu ausschließlich auf den Strukturbruch zurückzuführen. Der Effekt ist insbesondere wichtig für die Lohnungleichheit unter Frauen, da für sie der Teilzeitanteil viel höher und deshalb die Verzerrung vor 2010 stärker wirkt als für Männer.

Im Folgenden werden wir, wo es gekennzeichnet ist, die Korrekturmethode, welche wir in unserer Studie Fitzenberger und Seidlitz (2020) entwickelt haben, nutzen. Kurz gesprochen basiert diese auf einer Umgewichtung für die Jahre von 2000 bis 2011. Beschäftigungsverhältnisse, die als Vollzeit angegeben wurden, aber für die 
wir eine hohe Wahrscheinlichkeit errechnen, dass es sich um Teilzeitverhältnisse handelt, erhalten ein geringes Gewicht. Der Verzerrung wird so entgegen gewirkt.

In unserer Auswertung beschränken wir uns auf Beschäftigte zwischen 25 und 55 Jahren. Solche Beschränkungen sind in der Literatur üblich. Die meisten Studien beziehen sich ebenfalls nur auf eine Kernerwerbstätigkeitsphase mit ähnlichen Altersgrenzen. Ferner betrachten wir nur Westdeutschland (ohne Berlin). In den früheren Jahren war dies wichtig, um die Verläufe bis in die 1980er Jahre zurück zu zeichnen. Wir halten uns an dies, um die Vergleichbarkeit mit der Mehrzahl der Studien zu gewährleisten. ${ }^{5}$

Außerdem gewichten wir die Beschäftigungsverhältnisse nach ihrer Dauer. In den SIAB-Daten können grundsätzlich alle Beschäftigungsverhältnisse einem spezifischen Jahr zugeordnet werden. Erstrecken sie sich über das gesamte Jahr erhalten sie ein Gewicht von eins. Ansonsten entspricht es dem Quotienten aus der Anzahl der Tage und 365 bzw. 366, je nach Anzahl der Tage in dem betreffenden Jahr.

\subsection{Verlauf der Lohnungleichheit zwischen 2000 und 2017 mit und ohne Korrektur des Strukturbruchs von 2011}

Als erste Studie auf Basis der SIAB-Daten bis 2014 weist Möller (2016) darauf hin, dass der Anstieg der Lohnungleichheit seit 2011 gestoppt ist. Gleichzeitig zeigt seine Studie die deutlichen Auswirkungen des Strukturbruch in den SIAB-Daten auf die unteren Lohnquantile.

Zentral ist für uns die Frage, inwieweit sich die Korrektur des Strukturbruchs auf den Befund des Anstiegs der Lohnungleichheit bis 2010 auswirkt. Abb. 1 aus Bossler et al. (2020) zeigt die Lohnentwicklung für Männer und Frauen in Vollzeit seit dem Jahr 2000 ohne und mit Anwendung des Korrekturverfahrens von Fitzenberger und Seidlitz (2020). Wie vermutet steigt für Frauen die Lohnungleichheit bis 2010 mit der Korrektur etwas schwächer als in den Rohdaten. Für Männer zeigt sich hingegen, dass die Steigerung der Lohnungleichheit in den 2000er Jahren durch die Korrektur sogar größer wird. Die Notwendigkeit, den Strukturbruch zu korrigieren, wird deutlich angesichts des unplausibel starken Lohnwachstums für Frauen am 20\%-Quantil und am Median in den Jahren 2011 und 2012.

Weiter zeigt Abb. 1, dass spätestens ab 2012 die realen Vollzeitlöhne in Westdeutschland nach Korrektur an allen betrachten Quantilen kontinuierlich ansteigen. Für geringverdienende Frauen ist dieser Anstieg am stärksten. 2017 erreicht das 20\%-Quantil für Frauen fast wieder den Wert des Jahres 2000, während das 20\%Quantil für Männer 2017 immer noch 8 Logpunkte unterhalb des Wertes von 2000 liegt. Trotz des Reallohnwachstums ab 2012 für Männer und Frauen bleibt die Lohnungleichheit, gemessen an den Quantilsdifferenzen, auf konstant hohem Niveau.

\footnotetext{
5 Zu nennen wären etwa Dustmann et al. (2009); Card et al. (2013) und Antonczyk et al. (2018).
} 

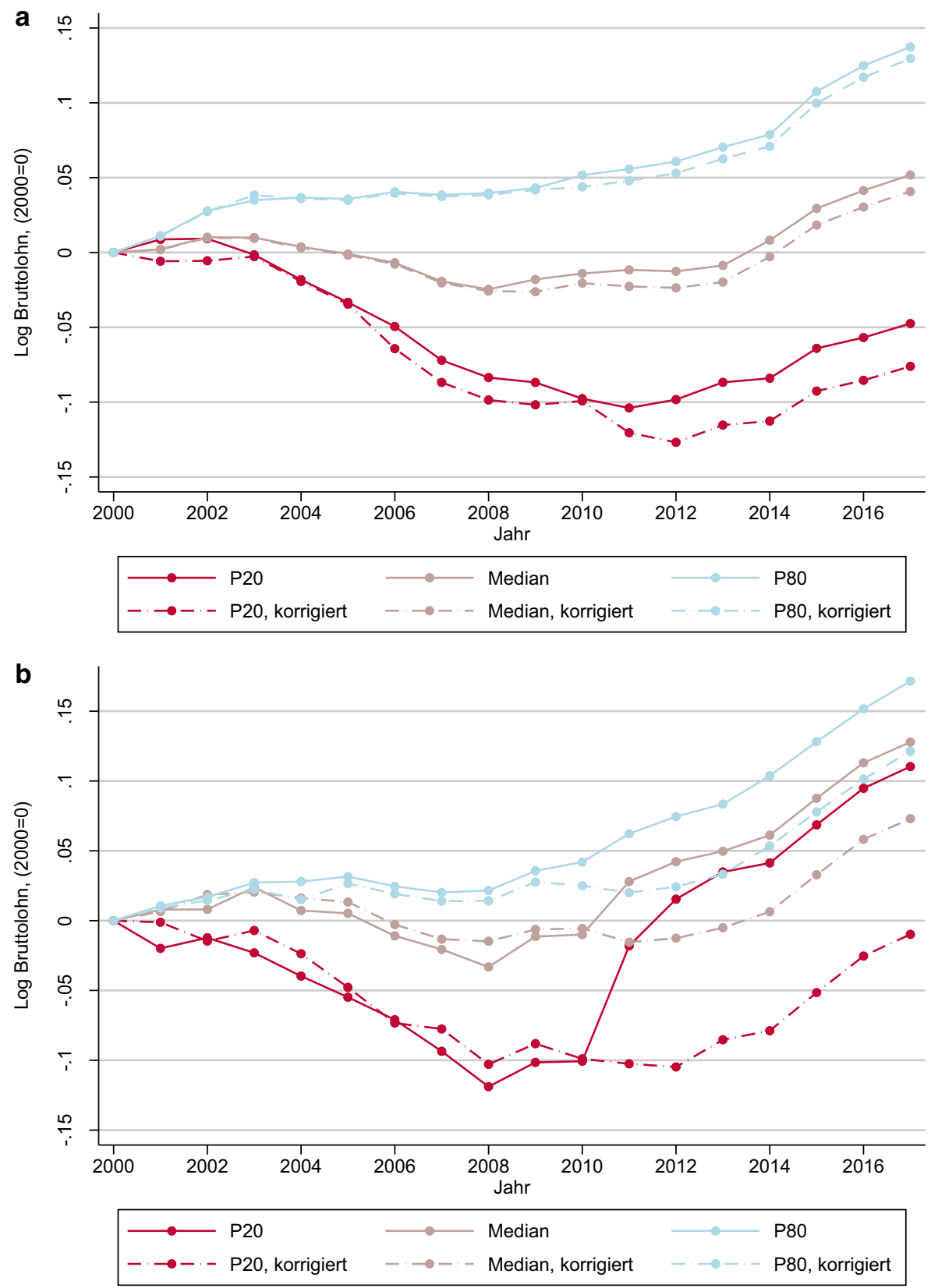

Abb. 1 Lohnentwicklung in Westdeutschland für Vollzeitbeschäftigte seit 2000, ohne und mit Korrektur des Strukturbruchs von 2011/2012. a Männer, b Frauen, Hinweise: Entwicklung der logarithmierten realen Tageslöhne an drei ausgewählten Quantilen (20\%-Quantil, Median, 80\%-Quantil), gemessen an der Veränderung gegenüber 2000. Die um den Strukturbruch bereinigten Reihen werden als ,korrigiert“ bezeichnet. Nominaler Tageslohn wird mit dem Konsumentenpreiseindex deflationiert. (Quelle: Bossler et al. 2020. Berechnung der Autoren in Anlehnung an Fitzenberger und Seidlitz 2020 auf Basis des SIAB7517) 


\section{Erklärungsfaktoren für die Entwicklung der Lohnungleichheit}

Dieser Abschnitt erörtert die Bedeutung von drei Faktoren für die Entwicklung der Lohnungleichheit seit 1990. Zu diesen Faktoren liegen aktuelle - und teils kontroverse - Befunde vor.

\subsection{Rückgang der Tarifbindung}

Die Tarifbindung ist in Deutschland in den letzten zwei Jahrzehnten stark zurückgegangen. Stattdessen handeln Firmen häufiger mit ihren einzelnen Beschäftigten die Entlohnung aus. Antonczyk et al. (2010, 2011) zeigen auf Basis der Verdienststrukturerhebungen des Statistischen Bundesamtes, dass allein im Zeitraum 2001 bis 2006 die Tarifbindung in den erfassten Wirtschaftszweigen der Privatwirtschaft um mehr als 16 Prozentpunkte abnahm. Ferner ist in den vergangenen Jahrzehnten auch der Anteil der Beschäftigten, die Mitglied in einer Gewerkschaft sind, massiv zurückgegangen (Fitzenberger et al. 2011).

Die Literatur geht davon aus, dass die Durchschnittslöhne im nicht tarifgebundenen Bereich niedriger und die Lohnstreuung höher als im tarifgebundenen Bereich des Arbeitsmarktes sind. Dieser Hypothese entsprechend legen die Befunde in Dustmann et al. (2009) und Biewen und Seckler (2020) nahe, dass der Rückgang der Tarifbindung ein wichtiger Treiber des Anstiegs der Lohnungleichheit in Westdeutschland bzw. Gesamtdeutschland war (siehe auch analoge Ergebnisse von Brüll und Gathmann 2019 für Ostdeutschland). Jedoch finden Antonczyk et al. (2010) für die Entwicklung der Ungleichheit im unteren Bereich der Lohnverteilung zwischen 2001 und 2006 keinen Beitrag des Rückgangs der Tarifbindung, wenn für firmenspezifische Effekte kontrolliert wird (ähnlich argumentieren Card et al. 2013).

Weiterhin zeigen Dustmann et al. (2014), dass der Anstieg der Lohnungleichheit zwischen 1995 und 2007 besonders stark im Bereich der tarifgebundenen Betriebe ausfiel. Die Studie führt dies u. a. darauf zurück, dass Abweichungen vom Tarifvertrag bei einer Zustimmung des Betriebsrats möglich sind, worin sich die Flexibilität des deutschen Systems industrieller Beziehungen zeige. Obwohl die deutsche Wirtschaft durch die Wiedervereinigung und die wirtschaftliche Öffnung der osteuropäischen Staaten unter starken Druck geriet, konnte diese Flexibilität den Anstieg der Arbeitslosigkeit begrenzen. Allerdings leistete sie auch einen erheblichen Beitrag zum Anstieg der Lohnungleichheit. Während Flächentarifverträge - und dadurch auch Gewerkschaften und Arbeitgeberverbände - an Einfluss verloren, wurde die lokale Entscheidungsebene, also Betriebsräte und vor allen Dingen Firmen, wichtiger. Zwischen 1995 und 2008 nahm der Anteil der Beschäftigten, die an Flächentarifverträge gebunden waren, von 75 auf 56 Prozent ab. Gleichzeitig blieb der Anteil der Beschäftigten mit einem Haustarifvertrag mit zehn Prozent in etwa konstant. Massiv stieg hingegen die Zahl derer an, die weder in der einen noch der anderen Form an einen Tarifvertrag gebunden waren.

Da es einen allgemeinen Mindestlohn erst ab 2015 gab, war der Lohnsetzung zumindest formell nach unten keine Grenze gesetzt. Die Löhne konnten so real über die Jahre sinken, was sie in der unteren Hälfte der Lohnverteilung bis zum Median von der Mitte der 1990er Jahre bis 2010 auch taten. Da zeitgleich die Löhne in 
der oberen Hälfte der Verteilung weiter stiegen, vergrößerte sich die Ungleichheit der Löhne. Allerdings sei daran erinnert, dass der Anstieg der Lohnungleichheit im tarifgebundenen Bereich aus den oben genannten Gründen noch stärker ausfiel.

Als Auslöser für den Anstieg der Lohnungleichheit erscheinen zwei Entwicklungen wichtig, zum einen die deutsche Wiedervereinigung und die wirtschaftliche Öffnung der östlichen Nachbarstaaten, wie Polen und Tschechien. Deutsche Firmen hatten neue Möglichkeiten, Arbeitsplätze ins Ausland zu verlagern, wo die Löhne unter dem Niveau in Deutschland lagen, was die Löhne hierzulande unter Druck setzte. Beides prägte den deutschen Arbeitsmarkt in den 1990er Jahren, sowohl für tarifgebundene wie auch für nicht tarifgebundene Firmen. Während die Nachwendeeffekte nachließen, blieb das Thema der Arbeitsplatzverlagerung bis in die 2000er Jahre auf der Tagesordnung und erhielt nach den EU-Osterweiterungen 2004 und 2007 neuen Auftrieb.

\subsection{Heterogenität in den Erwerbsbiographien}

In den letzten Jahrzehnten hat die Heterogenität der Berufskarrieren deutlich zugenommen. Vollzeitbeschäftigungsverhältnisse werden unterbrochen durch Phasen der Teilzeit- und Nichterwerbstätigkeit. Diese können sich auch dauerhaft negativ auf die Löhne auswirken. Die Erwerbsgeschichten bilden somit eine zusätzliche Ebene der Heterogenität. Weitere Faktoren wie die Alters- und Bildungsstruktur können sich verändern und einen Beitrag zu erhöhter Lohnungleichheit leisten (Biewen et al. 2018).

Auf dem deutschen Arbeitsmarkt gibt es seit Jahren eine wachsende Anzahl an Teilzeitbeschäftigten. Ihr Anteil an der Gesamtzahl der Beschäftigten stieg zwischen 2000 und 2014 von 3 auf 7 Prozent für Männer und von 33 auf 48 Prozent für Frauen (Fitzenberger und Seidlitz 2020).

Für unsere Analyse besonders relevant ist der Befund, dass auch der Anteil der Wechsler/innen zwischen den drei Bereichen Vollzeit-, Teilzeit- und Nichterwerbstätigkeit stieg. Bei Betrachtung der Vollzeiterwerbstätigen sinkt also der Anteil der Erwerbsbiographien, in denen in den vergangenen Jahren ausschließlich Vollzeit gearbeitet wurde. Dies ist politisch durchaus so gewollt und mit dem ,Teilzeit- und Befristungsgesetz“ von 2001 auch unterstützt worden (Biewen et al. 2018).

Für die Entlohnung der Beschäftigten ist die Erwerbsgeschichte eine wichtige Komponente. Phasen der Nichterwerbstätigkeit, die neben Arbeitslosigkeit auch alle freiwilligen Phasen ohne Erwerbstätigkeit (z.B. Erziehungszeiten) umfasst, wirken sich negativ auf die Entlohnung in zukünftigen Vollzeitjobs aus (Burda und Mertens 2001). Ebenfalls geringer entlohnt werden ehemalige Teilzeiterwerbstätige. Hätten diese stattdessen in Vollzeit gearbeitet, so könnten sie mit höheren Verdiensten in zukünftigen Arbeitsverhältnissen rechnen (Paul 2015).

Die Analyse in Biewen et al. (2018) zeigt für den Zeitraum bis 2010, dass für die Vollzeitbeschäftigten in der unteren Hälfte der Lohnverteilung die durchschnittliche Anzahl der Tage, die sie in den fünf Jahren zuvor in Nichterwerbstätigkeit verbracht haben, deutlich höher ist als für die Beschäftigten der oberen Hälfte. Sie haben auch im Durchschnitt etwas länger in Teilzeit gearbeitet als die gut Verdienenden, wobei der Unterschied hier geringer ist. Aufgrund dieser Unterschiede über die 
Lohnverteilung und der negativen Effekte von Teilzeit- und Nichterwerbstätigkeit auf die Verdienste in zukünftigen Vollzeitjobs ist die zunehmende Heterogenität in den Erwerbsbiographien ein Haupterklärungsfaktor für die gestiegene Lohnungleichheit unter den Vollzeitbeschäftigten zwischen 1985 und 2010. Nach den Ergebnissen in Biewen et al. (2018) können diese Veränderungen bei den Männern 14 bis 17 Prozent und bei den Frauen 17 bis 18 Prozent des Anstiegs der Lohnungleichheit erklären.

\subsection{Bedeutung der Arbeitsmarktlage für die Selektion von Erwerbstätigen}

Ein ebenfalls relevanter Faktor für die Entwicklung könnte die Lage am Arbeitsmarkt sein. Bei hoher Arbeitslosigkeit sind bestimmte Personen nicht mehr beschäftigt und ihre dann potentiellen Verdienste tauchen auch nicht mehr in der Lohnverteilung auf. Es ist naheliegend zu vermuten, dass bei einem Anstieg der Arbeitslosigkeit zunächst vor allem Beschäftigte mit niedrigen Bildungsabschlüssen und geringer Berufserfahrung, also tendenziell Geringverdienende, ihre Arbeit verlieren. Dann würde ein Anstieg der Arbeitslosigkeit die Lohnungleichheit verringern, während ein Rückgang der Arbeitslosigkeit sie erhöhen müsste.

Fitzenberger und de Lazzer (2020) untersuchen, ob die Selektion in die Erwerbstätigkeit zum Anstieg der Ungleichheit beigetragen hat. Nach den Ergebnissen dieser Studie wäre die Lohnungleichheit in einem hypothetischen Szenario mit Vollbeschäftigung höher als in einem Arbeitsmarkt mit Arbeitslosigkeit. Dies würde vor allem die Ungleichheit im unteren Bereich der Lohnverteilung betreffen. Das Ergebnis spiegelt die positive Selektion der Beschäftigten in einem Arbeitsmarkt mit Arbeitslosigkeit wider.

Hinsichtlich der Veränderung der Selektion der Beschäftigten im Zeitverlauf ergibt sich aus den Ergebnissen von Fitzenberger und de Lazzer (2020), dass die Ungleichheit 2010 mit der Selektion der Erwerbstätigen von 1995 höher gewesen wäre als die tatsächlich in 2010 beobachtete Ungleichheit. Dies impliziert, dass sich die Selektion der Beschäftigten im Jahr 2010 gegenüber 1995 in einer lohnungleichheitsreduzierenden Weise verbessert hat. Somit kann die Hypothese verworfen werden, dass der Rückgang der Arbeitslosigkeit vor allem die Beschäftigung von negativ selektierten Beschäftigten erhöht habe, was mechanisch den Anstieg der Lohnungleichheit erklärt hätte. Diese Ergebnisse sind jedoch als sehr vorläufig einzustufen und bedürfen weiterer Überprüfung in zukünftigen Forschungsarbeiten.

\section{Schlussfolgerungen und Ausblick}

Dieser Beitrag dokumentiert und interpretiert in einem Rückblick und Überblick der Literatur die Entwicklung der Lohnungleichheit in Deutschland und geht auf mögliche Datenprobleme ein. Der Fokus liegt auf der Entwicklung der Lohnungleichheit für Vollzeitbeschäftigte in Westdeutschland. Die meisten Befunde basieren auf den Daten der Stichprobe der Integrierten Erwerbsbiographien, den SIAB-Daten, welche einen Strukturbruch in der Erfassung von Vollzeitbeschäftigung aufweisen, dem in der Analyse Rechnung getragen werden muss (Möller 2016; Fitzenberger und 
Seidlitz 2020). Viele Studien betrachten dabei ausschließlich westdeutsche Männer. Wir haben uns bemüht, den Beitrag um ausgewählte Ergebnisse für Frauen und für Ost- oder Gesamtdeutschland zu ergänzen.

Zentrale Befunde sind: In Westdeutschland kam es zwischen 1980 und 2010 zu einem deutlichen Anstieg der Lohnungleichheit von Vollzeitbeschäftigten, der sich zunächst auf den oberen Bereich der Lohnverteilung beschränkte und ab Mitte der 1990er Jahre sowohl im oberen als auch im unteren Bereich der Lohnverteilung fortsetzte. Im Zeitraum 1995 bis 2010 ging die Entwicklung mit starken Reallohnverlusten im unteren Bereich der Lohnverteilung einher. Nach 2010 stieg die Lohnungleichheit für Vollzeitbeschäftigte nicht weiter an, aber es kam zu keinem deutlichen Rückgang der Lohnungleichheit. Die Reallöhne stiegen über die gesamte Lohnverteilung seit 2010 deutlich an.

Die zitierten Studien sehen in dem zunehmenden internationalen Handel (,Globalisierung') keinen Erklärungsfaktor für den Anstieg der Lohnungleichheit bis 2010. Während der Mindestlohn in Westdeutschland zu keinem deutlichen Rückgang der Lohnungleichheit führt, lässt sich ein deutlicher ungleichheitsreduzierender Effekt für Ostdeutschland feststellen.

Kontrovers wird in der Literatur der Beitrag des Rückgangs der Tarifbindung für die Lohnungleichheit von Vollzeitbeschäftigten in Westdeutschland diskutiert. Es gibt erste Hinweise, dass die im Zeitverlauf sich verändernde Selektion von Beschäftigten im Hinblick auf Erwerbsunterbrechungen und Phasen der Teilzeiterwerbstätigkeit ein weiterer Erklärungsfaktor für den Anstieg der Lohnungleichheit von Vollzeitbeschäftigten darstellt. Noch sehr vorläufig ist die Analyse des Einflusses der veränderten Selektion der Vollzeitbeschäftigten durch die verbesserte Arbeitsmarktlage im Hinblick auf unbeobachtete Charakteristika. Erste vorläufige Ergebnisse auf Basis der Studie Fitzenberger und de Lazzer (2020) zeigen, dass sich die Selektion der Beschäftigten im Jahr 2010 gegenüber 1995 in einer lohnungleichheitsreduzierenden Weise verbessert hat.

Einschränkend ist darauf hinzuweisen, dass verschiedene wichtige Aspekte in diesem Beitrag nur unzureichend oder gar nicht behandelt werden konnten. $\mathrm{Zu}$ nennen sind hier die Lohnunterschiede zwischen Männern und Frauen und die Unterschiede in den Stundenlöhnen zwischen Vollzeit- und Teilzeitbeschäftigten, wobei beide Aspekte stark miteinander zusammenhängen. Des Weiteren sind die Unterschiede in der Entwicklung der Lohnungleichheit zwischen Ost- und Westdeutschland, die Marktmacht von Unternehmen und die Migration nach Deutschland zu nennen ( siehe zu den beiden letzten Punkten u. a. die Diskussion in Card et al. (2013).

Funding Open Access funding provided by Projekt DEAL.

Open Access Dieser Artikel wird unter der Creative Commons Namensnennung 4.0 International Lizenz veröffentlicht, welche die Nutzung, Vervielfältigung, Bearbeitung, Verbreitung und Wiedergabe in jeglichem Medium und Format erlaubt, sofern Sie den/die ursprünglichen Autor(en) und die Quelle ordnungsgemäß nennen, einen Link zur Creative Commons Lizenz beifügen und angeben, ob Änderungen vorgenommen wurden.

Die in diesem Artikel enthaltenen Bilder und sonstiges Drittmaterial unterliegen ebenfalls der genannten Creative Commons Lizenz, sofern sich aus der Abbildungslegende nichts anderes ergibt. Sofern das betreffende Material nicht unter der genannten Creative Commons Lizenz steht und die betreffende Handlung 
nicht nach gesetzlichen Vorschriften erlaubt ist, ist für die oben aufgeführten Weiterverwendungen des Materials die Einwilligung des jeweiligen Rechteinhabers einzuholen.

Weitere Details zur Lizenz entnehmen Sie bitte der Lizenzinformation auf http://creativecommons.org/ licenses/by/4.0/deed.de.

\section{Literatur}

Acemoglu D, Autor D (2011) Skills, tasks, and technologies: implications for employment and earnings. In: Ashenfelter O, Card D (Hrsg) Handbook of labor economics, Bd. 4B, S 1043-1171

Antonczyk D, Fitzenberger B, Leuschner U (2009) Can a task-based approach explain the recent changes in the German wage structure? J Econ Stat 229(2+3):214-238

Antonczyk D, Fitzenberger B, Sommerfeld K (2010) Rising wage inequality, the decline of collective bargaining, and the gender wage gap. Labour Econ 17(5):835-847

Antonczyk D, Fitzenberger B, Sommerfeld K (2011) Anstieg der Lohnungleichheit, Rückgang der Tarifbindung und Polarisierung. Z ArbeitsmarktForsch 44(1/2):15-27

Antonczyk D, DeLeire T, Fitzenberger B (2018) Polarization and rising wage inequality: comparing the U.S. and Germany. Econometrics 6:20

Antoni M, Ganzer A, vom Berge P (2019) Stichprobe der Integrierten Arbeitsmarktbiografien Regionalfile (SIAB-R) 1975-2017 (FDZ-Datenreport, 04/2019 (de))

Baumgarten D, Felbermayr G, Lehwald S (2020) Dissecting between-plant and within-plant wage dispersion: evidence from Germany. Ind Relat 59:85-122

Biewen M, Seckler M (2020) Unions, internationalization, tasks, firms, and worker characteristics: a detailed decomposition analysis of rising wage inequality in Germany. J Econ Inequal 17:461-498

Biewen M, Fitzenberger B, de Lazzer J (2018) The role of employment interruptions and part-time work for the rise in wage inequality. IZA J Labor Econ. https://doi.org/10.1186/s40172-018-0070-y

Bossler M, Schank T (2020) Wage inequality in Germany after the minimum wage introduction. IZADiscussion Paper, Bd. 13003

Bossler M, Fitzenberger B, Seidlitz A (2020) Neues zur Lohnungleichheit in Deutschland. ifo Schnelldienst, Bd. 73(1)

Brüll E, Gathmann C (2019) Evolution of wage inequality in East Germany. Chapter 3 of Dissertation of Eduard Brüll, University of Heidelberg. archiv.ub.uni-heidelberg.de/volltextserver/26155/. Zugegriffen: 10 . Mai 2020

Burda M, Mertens A (2001) Estimating wage losses of displaced workers in Germany. Labour Econ $8: 15-41$

Card D, Heining J, Kline P (2013) Workplace heterogeneity and the rise of German wage inequality. Q J Econ 128(3): $967-1015$

Dustmann C, Fitzenberger B, Schönberg U, Spitz-Oener A (2014) From sick man of Europe to economic superstar: Germany's resurgent economy. J Econ Perspect 28(1):167-188

Dustmann C, Ludsteck J, Schönberg U (2009) Revisiting the German wage structure. Q J Econ 124(2):843-881

Fitzenberger B (1999) Wages and employment across skill groups: an analysis for West Germany. Physica, Heidelberg

Fitzenberger B (2012) Expertise zur Entwicklung der Lohnungleichheit in Deutschland, Sachverständigenrat zur Begutachtung der gesamtwirtschaftlichen Entwicklung, Arbeitspapier 4/2012. https://www. econstor.eu/handle/10419/74750. Zugegriffen: 10. Mai 2020

Fitzenberger B, de Lazzer J (2020) Changing selection into full-time work and its effect on wage inequality in Germany. Unveröffentlichtes Manuskript, Humboldt-Universität zu Berlin

Fitzenberger B, Seidlitz A (2020) The 2011 break in the part-time indicator and the evolution of wage inequality in Germany. J Labour Market Res. https://doi.org/10.1186/s12651-019-0265-0

Fitzenberger B, Kohn K, Wang Q (2011) The erosion of union membership in Germany: determinants, densities, decompositions. J Popul Econ 24(1):141-165

Gernandt J, Pfeiffer F (2007) Rising wage inequality in Germany. Jahrb Natl Okon Stat 227(4):358-380

Goldschmidt D, Schmieder J (2017) The rise of domestic outsourcing and the evolution of the German wage structure. Q J Econ 132(3):1165-1217

Heckman J (2002) Flexibility and job creation: lessons for Germany. NBER Working Paper, Bd. 9194

Katz L, Autor D (1999) Changes in the wage structure and earnings inequality. In: Ashenfelter O, Card D (Hrsg) Handbook of labor economics, Bd. 3A. Elsevier, Amsterdam, S 1463-1555 
Machin S, Van Reenen J (2008) Changes in wage inequality. In: Durlauf SN, Blume LE (Hrsg) The new Palgrave dictionary of economics, 2. Aufl.

Möller J (2016) Lohnungleichheit - Gibt es eine Trendwende? Wirtschaftsdienst 96(1):38-44

Paul M (2015) Is there a causal effect of working part-time on current and future wages? Scand J Econ 118:494-523

Prasad ES (2004) The unbearable stability of the German wage structure: evidence and interpretation. IMF Staff Papers, Bd. 51, S 354-385

Riphahn RT, Schnitzlein DD (2016) Wage mobility in East and West Germany. Labour Econ 39:11-34

Spitz-Oener A (2006) Technical change, job tasks, and rising educational demands: looking outside the wage structure. J Labor Econ 24(2):235-270

Hinweis des Verlags Der Verlag bleibt in Hinblick auf geografische Zuordnungen und Gebietsbezeichnungen in veröffentlichten Karten und Institutsadressen neutral. 